\title{
Glia- and neuron-specific functions of TrkB signalling during retinal degeneration and regeneration
}

\author{
Chikako Harada1,2, Xiaoli Guo', Kazuhiko Namekata1, Atsuko Kimura', Kazuaki Nakamura1', Kohichi Tanaka², \\ Luis F. Parada² \& Takayuki Harada1,2
}

Glia, the support cells of the central nervous system, have recently attracted considerable attention both as mediators of neural cell survival and as sources of neural regeneration. To further elucidate the role of glial and neural cells in neurodegeneration, we generated TrkB ${ }^{\text {GFAP }}$ and TrkB ${ }^{c-k i t}$ knockout mice in which TrkB, a receptor for brain-derived neurotrophic factor (BDNF), is deleted in retinal glia or inner retinal neurons, respectively. Here, we show that the extent of glutamate-induced retinal degeneration was similar in these two mutant mice. Furthermore in TrkB GFAP knockout mice, BDNF did not prevent photoreceptor degeneration and failed to stimulate Müller glial cell proliferation and expression of neural markers in the degenerating retina. These results demonstrate that BDNF signalling in glia has important roles in neural protection and regeneration, particularly in conversion of Müller glia to photoreceptors. In addition, our genetic models provide a system in which glia- and neuronspecific gene functions can be tested in central nervous system tissues in vivo.

\footnotetext{
${ }^{1}$ Department of Molecular Neurobiology, Tokyo Metropolitan Institute for Neuroscience, 2-6 Musashidai, Fuchu, Tokyo 183-8526, Japan. ${ }^{2}$ Department of Developmental Biology and Kent Waldrep Foundation Center for Basic Research on Nerve Growth and Regeneration, University of Texas Southwestern Medical Center, 6000 Harry Hines Boulevard, Dallas, Texas 75390-9133, USA. ${ }^{3}$ Laboratory of Molecular Neuroscience, School of Biomedical Science and Medical Research Institute, Tokyo Medical and Dental University, Tokyo 113-8510, Japan. Correspondence and requests for materials should be addressed to L.F.P. (email: luis.parada@utsouthwestern.edu) or to T.H. (email: harada-tk@igakuken.or.jp).
} 
$\mathrm{n}$ the central nervous system (CNS), glial cells have long been thought to simply provide scaffolding and physical support for neurons, whose electrical impulses underlie all sensation, movement and thought. However, recent studies have demonstrated that glia, which account for $90 \%$ of the cells in some regions of the human brain, have more significant roles than previously hypothesized $^{1}$. For example, glial cells guide migrating neurons to their destinations, control synapse number and may participate in the homeostatic activity-dependent regulation of synaptic connectivity $^{2,3}$. In addition, radial glial cells, which express a number of markers also expressed by neural progenitors, were demonstrated to dedifferentiate, proliferate and give rise to neurons in vivo ${ }^{4-6}$. We previously demonstrated that the glutamate transporter GLAST, one such progenitor marker ${ }^{7}$, is expressed in retina-specific Müller glial cells, which are thought to carry out many of the functions of radial glia, astrocytes and oligodendrocytes in the $\mathrm{CNS}^{8,9}$. Müller glial cells dedifferentiate in response to neurotoxic damage or injury, implying that even the adult mammalian retina may have regenerative potential $^{10,11}$. The response of Müller glia to acute retinal damage may be mediated by growth factors produced by retinal cells, such as basic fibroblast growth factor (bFGF) and brain-derived neurotrophic factor $(\mathrm{BDNF})^{12-14}$. BDNF is known to regulate neural cell survival and axonal outgrowth mainly by activating TrkB receptors, which stimulates various signalling cascades, such as mitogen-activated protein kinase pathway, phosphatidylinositol 3-kinase pathway and Fyn-mediated actin polymerization ${ }^{15-17}$. However, TrkB expression is low in photoreceptors relative to other retinal cell types, including retinal ganglion cells (RGCs) and Müller glia ${ }^{12,13,18}$. Consequently, we previously proposed a model in which exogenously applied or microglia-derived neurotrophins regulate photoreceptor survival indirectly by regulating secondary trophic factor production in Müller glia ${ }^{12,19}$. However, the effects that such a glia-neuron interaction would have in vivo or whether it would be relevant for the protection of neural cell types that express TrkB remained unclear.

In the present study, we prepared two conditional knockout (KO) mice in which TrkB was deleted from retinal glia or from two types of retinal neurons (RGCs and amacrine cells). These mice enabled us to separately examine direct and indirect effects of TrkB on neuroprotection. We demonstrate that TrkB signalling in Müller glia is critically involved in neural protection and regeneration during retinal degeneration. These mice may enable the further study of genes involved in the glia-neuron network and neurodegeneration.

\section{Results}

Glia- and neuron-specific TrkB ablation in the retina. Previous studies have shown that the GFAP-Cre transgenic strain, in which Cre expression is regulated by the human GFAP promoter, expresses Cre recombinase not only in mature astrocytes but also in multipotent radial glial cells that exhibit neural stem/progenitor cell properties $^{20,21}$. To examine expression of the GFAP-Cre transgene in the adult retina, we crossed GFAP-Cre mice with a Rosa26LacZ reporter line $e^{22,23}$ in which recombination results in expression of $\beta$-galactosidase ( $\beta$-gal; Fig. 1a,b). To identify the specific $\beta$-gal immunopositive (IP) cell type(s), we carried out co-staining with markers of other retinal lineages. $\beta$-gal-IP cells were double labelled with glutamine synthetase (GS; a marker of Müller glial cells), but not with calretinin (a marker of RGCs and amacrine cells), calbindin (a marker of horizontal cells) or protein kinase C (PKC; a marker of bipolar cells; Fig. 1b). These results revealed that $\beta$-gal expression, which reflects Cre recombinase expression, is restricted to glial cells in GFAP-Cre LacZ mice. On the other hand, in $c$-kit-Cre LacZ mice ${ }^{24}, \beta$-gal-IP cells were double labelled with calretinin and TUJ1 (another RGC marker), but not with GS, calbindin or PKC (Fig. 1b). These results indicate that, in contrast to GFAP-Cre LacZ mice, expression of Cre recombinase is restricted to RGCs and amacrine cells in $c$-kit-Cre LacZ mice (Fig. 1a).
To selectively eliminate TrkB from retinal glia or neurons, we next crossed TrkB flox/flox mice ${ }^{25}$ with GFAP-Cre or $c$-kit-Cre mice. The resulting double transgenic $\operatorname{TrkB}^{\text {flox/flox }} ; G F A P-C r e+$ and TrkB $B^{\text {flox/flox}}$; $c$-kit-Cre + mice were termed as $\operatorname{TrkB}^{\mathrm{GFAP}} \mathrm{KO}$ mice and $\operatorname{TrkB} \mathrm{B}^{\mathrm{c}-\mathrm{kit}} \mathrm{KO}$ mice, respectively. Retinal development and structure were normal in both strains compared with wild-type (WT) mice (Supplementary Fig. S1). Immunohistochemical analysis revealed that TrkB protein expression in Müller glia (arrowheads in Fig. 1c) was eliminated in TrkB ${ }^{\mathrm{GFAP}} \mathrm{KO}$ mice. In contrast, TrkB expression was eliminated in RGCs (arrowheads in Fig. 1d) and amacrine cells (arrows in Fig. 1d) in TrkB ${ }^{c-k i t} \mathrm{KO}$ mice. Taken together, these findings demonstrate that GFAP-Cre and $c$-kit-Cre virtually eliminated TrkB expression from retinal glia and neurons, respectively.

Direct and indirect effects of BDNF on RGC protection. Neurotrophins prevent RGC death from glutamate neurotoxicity ${ }^{26}$. As RGCs express TrkB receptors, we compared the roles of TrkB signalling in RGCs and Müller glia in BDNF-dependent neuroprotection to determine the direct and indirect effects, respectively. For this purpose, we prepared retinal explants from $\mathrm{WT}, \operatorname{TrkB}^{\mathrm{c}-\mathrm{kit}} \mathrm{KO}$ and TrkB ${ }^{\text {GFAP }}$ KO mice (Fig. 2a). The WT retinal explants stimulated with $5 \mathrm{mM}$ glutamate for $1 \mathrm{~h}$ showed a decrease in the number of NeuNpositive neurons in the ganglion cell layer (GCL) compared with the untreated control explants (Fig. 2a,b). As expected, the same treatment significantly decreased the number of surviving RGCs in the $\mathrm{TrkB}^{\mathrm{c}-\mathrm{kit}} \mathrm{KO}$ mouse retina. We predicted milder RGC degeneration in the $\operatorname{TrkB}^{\mathrm{GFAP}} \mathrm{KO}$ compared with the $\operatorname{TrkB}^{\mathrm{c}-\mathrm{kit}} \mathrm{KO}$ mouse retina; however, RGC loss was as severe as that observed in the $\operatorname{TrkB}^{\mathrm{c}-\mathrm{kit}} \mathrm{KO}$ mouse retina (Fig. 2a,b). These results suggest that, at least under our experimental conditions, the indirect protective effect of BDNF by Müller glia is as powerful as the direct effect through TrkB in RGCs.

To further examine the function of TrkB signalling in retinal glia, we next prepared cultured Müller cells from WT and TrkB ${ }^{\text {GFAP }}$ $\mathrm{KO}$ mice and analysed them by western blot. Consistent with the immunohistochemical results (Fig. 1c), TrkB protein expression in Müller glia was not detectable in TrkB ${ }^{\text {GFAP }}$ KO mice (Fig. 3a). We next examined whether exogenous BDNF stimulates TrkB-dependent signal transduction in Müller glia by observing the activation of cyclic AMP response element-binding (CREB) protein. In WT Müller cells, the basal phosphorylated CREB (pCREB) expression level was very low, but BDNF clearly increased the ratio of pCREB to CREB (Fig. 3b). In contrast, the effect of BDNF on CREB activation was only marginal in TrkB ${ }^{\text {GFAP }}$ KO Müller cells (Fig. 3b). We also examined the effect of BDNF on CREB phosphorylation in vivo. In WT retinas, intraocular injection of BDNF induced pCREB expression in RGCs and Müller glia (arrow and arrowhead in Fig. 3c). In TrkB ${ }^{\text {GFAP }} \mathrm{KO}$ mice, BDNF-induced pCREB expression in RGCs was comparable with WT retinas, but expression was barely detectable in Müller glia (Fig. 3c). These results confirmed a lack of TrkB-dependent signal transduction in Müller glia in $\operatorname{TrkB}^{\text {GFAP }} \mathrm{KO}$ mice. We previously demonstrated that treatment of cultured rat Müller cells with BDNF increases the production of several trophic factors that can protect retinal neurons ${ }^{19}$. Similarly, in mouse Müller cells, BDNF increased mRNA expression levels of BDNF, bFGF, ciliary neurotrophic factor and glial cell line-derived neurotrophic factor (Fig. 3d). However, BDNF failed to stimulate the production of trophic factors in Müller cells prepared from TrkB ${ }^{\text {GFAP }} \mathrm{KO}$ mice (Fig. 3d). Thus, TrkB elimination in Müller glia impairs the transcription of trophic factors, which may lead to RGC vulnerability during glutamate neurotoxicity.

Accelerated photoreceptor degeneration in TrkB ${ }^{\mathrm{GFAP}} \mathrm{KO}$. Various trophic factors rescue photoreceptors during retinal degeneration $^{27-29}$. As photoreceptors do not express a high level of neurotrophin receptors, the indirect glia-mediated rescue pathway may be more important for photoreceptors than for other cell types, 


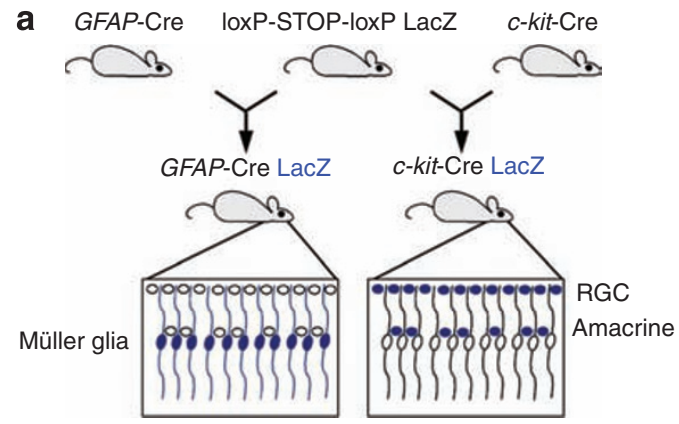

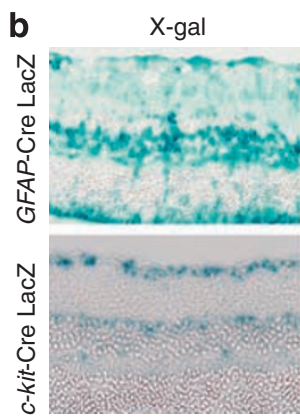

C

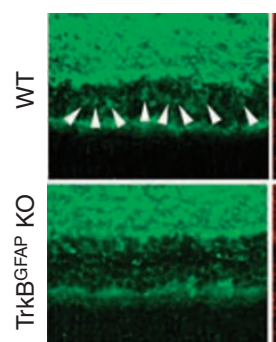

$\beta$-gal / GS
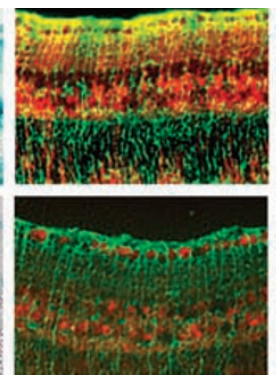

GS

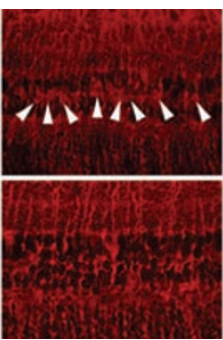

$\beta$-gal / Calretinin
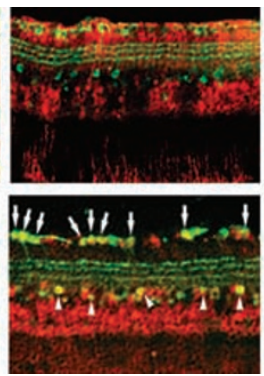

Merge

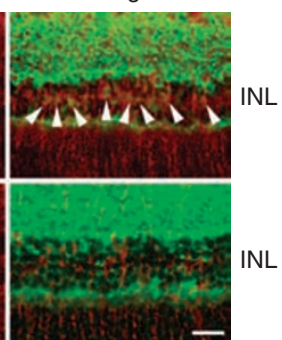

$\beta$-gal / Calbindin
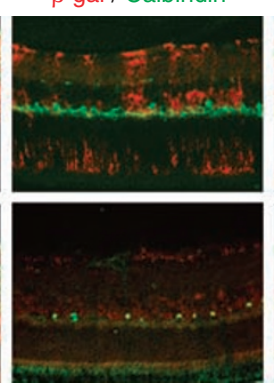

d

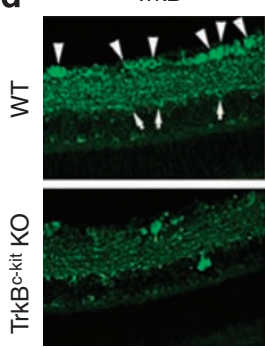

$\beta$-gal / PKC

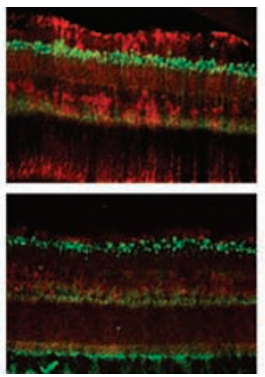

Calretinin

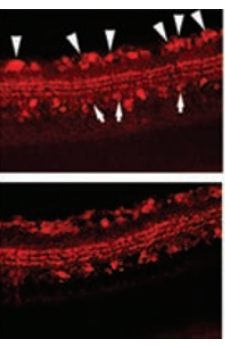

$\beta$-gal / TUJ1

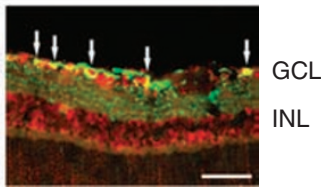

Merge

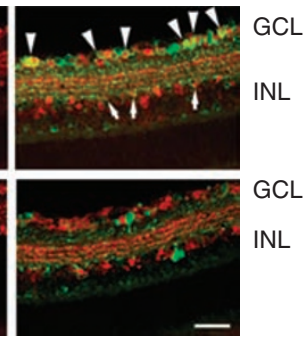

Figure 1 | Establishment of glia- and neuron-specific TrkB conditional KO mice. (a) Schematic diagram of transgenically targeted fate-mapping strategy. GFAP-Cre and c-kit-Cre mice were crossed with Rosa26-LacZ reporter mice. In Cre reporter offspring, only cells that express Cre excise a loxP-flanked stop signal and activate constitutive LacZ expression. Blue colour depicts LacZ-positive cells. (b) X-gal staining (blue) and immunostaining (red) of $\beta$-gal-positive cells. Glutamine synthetase (GS), calretinin, calbindin, protein kinase C (PKC) and TUJ1 were used as cell type-specific markers (green). Overlapping immunoreactivities (yellow) of $\beta$-gal and GS, calretinin or TUJ1 indicate that Cre-mediated recombination occurs in Müller glial cells in GFAP-Cre LacZ mice, and RGCs (arrows) and amacrine cells (arrowheads) in c-kit-Cre LacZ mice. (c) Immunohistochemical analysis of TrkB (green) and GS (red) showed loss of TrkB from Müller glial cells (arrowheads) in TrkBGFAP KO mice. (d) Immunohistochemical analysis of TrkB (green) and calretinin (red) showed loss of TrkB from RGCs (arrowheads) and amacrine cells (arrows) in TrkBc-kit KO mice. Scale bar, $100 \mu \mathrm{m}$. GCL, ganglion cell layer; INL, inner nuclear layer; RGC, retinal ganglion cell.

including RGCs ${ }^{12,13,18}$. To further elucidate the effect of the glianeuron network in neuroprotection in vivo, we used a second animal disease model, $\mathrm{N}$-methyl- $\mathrm{N}$-nitrosourea (MNU)-induced photoreceptor degeneration (Fig. 4a). MNU is an alkylating agent that causes DNA methylation and activates caspases, leading to photoreceptor apoptosis in various animal species ${ }^{30}$. In WT mice, $15 \mathrm{mg} \mathrm{kg}^{-1} \mathrm{MNU}$ decreased the thickness of the outer nuclear layer (ONL), but intravitreous injection of BDNF partially rescued the photoreceptor loss (Fig. 4b,c). However, in TrkB ${ }^{\mathrm{GFAP}} \mathrm{KO}$ mice, $7.5 \mathrm{mg} \mathrm{kg}^{-1} \mathrm{MNU}$ resulted in an $\sim 50 \%$ loss of the ONL, which was significantly greater than the loss in WT retinas, and BDNF failed to induce photoreceptor survival against any doses of MNU (Fig. 4b,c). The protective effect of BDNF in WT mice diminished in the presence of a higher dose of MNU ( $60 \mathrm{mg} \mathrm{kg}^{-1}$ ); this dose completely abolished the ONL but left the inner retinal layer (IRL) intact (Fig. 4b,d). In contrast, the same dose of MNU applied to TrkB ${ }^{\mathrm{GFAP}} \mathrm{KO}$ mice induced considerable loss of the IRL as well as the removal of the ONL (Fig. 4b,d). These in vivo data suggest that glial TrkB signalling has a pivotal role in the protection of surrounding neurons.
Regenerative capacity of Müller glia through TrkB signalling. Recent studies have provided evidence that radial glia sustain neural regeneration even in the adult brain ${ }^{4-6}$. Müller glia are considered to be a type of radial glia that may function as retinal progenitor cells $^{10,11}$. We therefore examined the regenerative response of Müller cells to BDNF in the MNU-induced photoreceptor degeneration model (Fig. 5a). To observe dividing cells, we labelled WT and TrkB GFAP KO animals with 5-bromodeoxyuridin (BrdU). Although few BrdU-labelled cells were detected at day 5, intraocular injection of BDNF clearly increased BrdU-labelling in GLAST-IP cells in the inner retina (arrows) and in some cells in the ONL (Fig. 5b). In contrast, BDNF injection did not promote BrdU incorporation in TrkB ${ }^{\text {GFAP }}$ KO mice or WT mice treated with K252a, an inhibitor of Trk receptors (Fig. 5b). At day 7, BDNF-induced BrdU-positive cells were detected predominantly in the remaining ONL, where they were co-labelled with rod photoreceptor markers, such as rhodopsin and recoverin (Fig. 5c). However, BDNF-induced BrdU incorporation was suppressed in TrkB ${ }^{\mathrm{GFAP}} \mathrm{KO}$ mice (Supplementary Fig. S2). Furthermore, these BrdU-labelled cells were overlapped with $\beta$-gal 
a
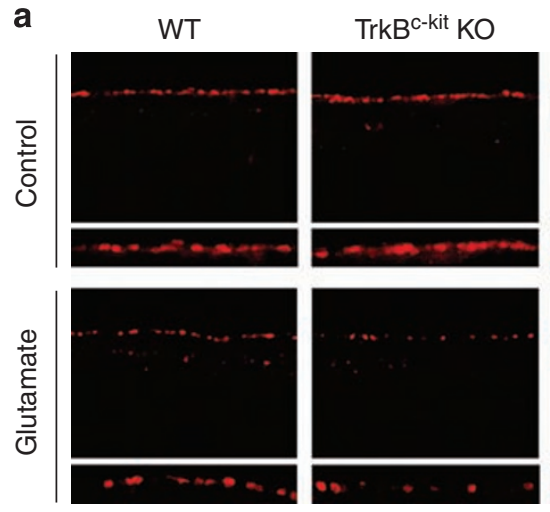

TrkB GFAP KO

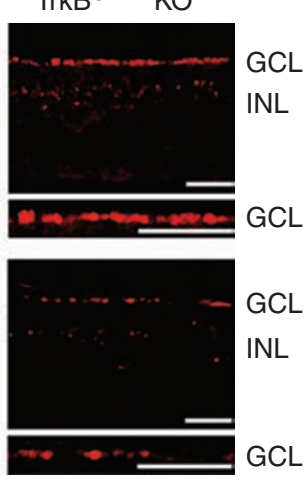

b

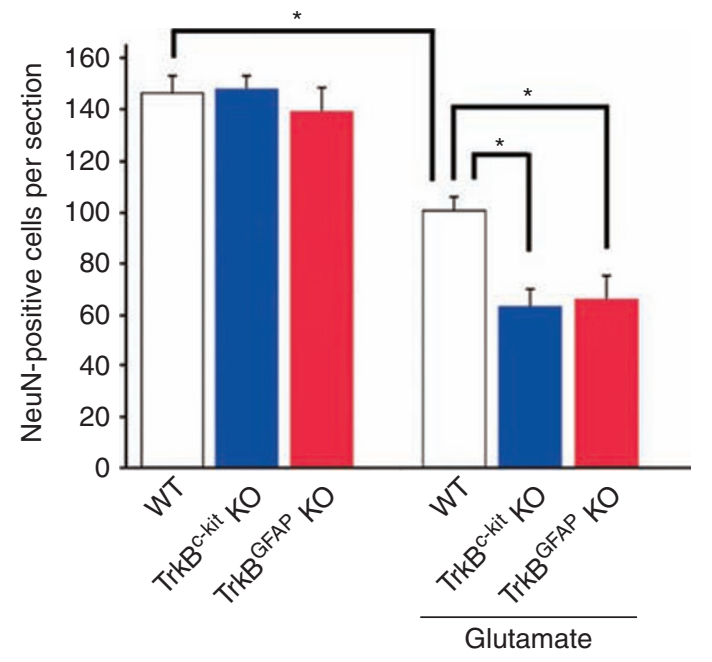

Figure 2 | Similar susceptibility to glutamate neurotoxicity in TrkB $^{\text {c-kit }}$ KO and TrkB ${ }^{\text {GFAP }} \mathbf{K O}$ retinas. (a) Immunohistochemical analysis of mouse retinal explants from $W T, T r k B^{c-k i t} K O$ and TrkB ${ }^{\text {GFAP }} K O$ mice stained with an anti-NeuN antibody. Explants were untreated (control) or treated with $5 \mathrm{mM}$ glutamate for $1 \mathrm{~h}$. Scale bar, $50 \mu \mathrm{m}$. (b) Quantification of NeuNpositive cells in the GCL. Data are shown as the mean +s.e.m. $(n=6)$. ${ }^{\star} P<0.01$. GCL, ganglion cell layer; INL, inner nuclear layer.

staining in GFAP-Cre LacZ mice, providing evidence that they were derived from Müller glia (Fig. 5c). In addition, some BrdU-positive cells were detected in the inner retina and double labelled with Brn3b (another RGC marker) or calretinin (Fig. 5d). These results suggest the possibility that BDNF can convert the proliferating Müller glia to rod photoreceptors and, to a lesser extent, to other retinal neurons in the MNU model. Thus, TrkB deficiency achieved by Cre-mediated recombination results in the loss of regenerating Müller cells.

We further examined the effects of BDNF on cultured Müller cells and found increased nestin expression, a neural stem/progenitor cell marker, in WT but not in TrkB ${ }^{\text {GFAP }}$ KO Müller cells (Fig. 6a). When neural stem cells are induced to differentiate into the neurogenic pathway, Mash1, a proneural gene, is derepressed through a signalling cascade that depends on expression of the calcium/calmodulindependent protein kinase II $\delta$ (CaMKII $\delta)^{31,32}$. In the retina, Mash1 is involved in the generation of bipolar cells and photoreceptors from progenitor cells ${ }^{33,34}$. A previous study showed that CaMKII $\delta$ mediates the phosphorylation of Hes1, which is required for Mash1 activation $^{31}$. As BDNF can stimulate CaMKII through $\operatorname{TrkB}^{16}$, we next examined whether BDNF regulates this pathway in neural regeneration. We found that BDNF increased Mash1 levels in WT but not in TrkB ${ }^{\mathrm{GFAP}} \mathrm{KO}$ Müller cells, and the subsequent quantitative analysis revealed that BDNF had no effect on Hes1 expression
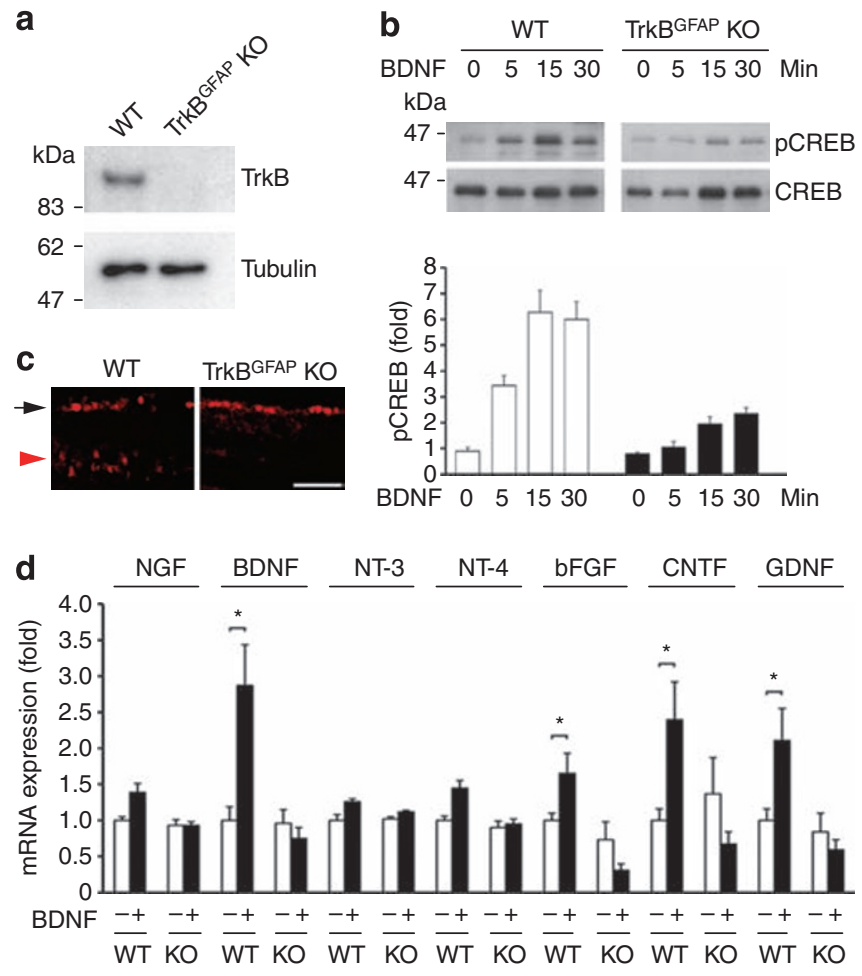

Figure 3 | Functional analysis of Müller glial cells in TrkB ${ }^{\text {GFAP }}$ KO mice. (a) Immunoblot analysis of TrkB protein in cultured Müller cells from WT and TrkB GFAP KO mice. (b) Immunoblot analysis of phosphorylated CREB ( $p C R E B$ ) and total CREB in cultured Müller cells from WT and TrkB ${ }^{\text {GFAP }} \mathrm{KO}$ mice. BDNF (50 $\mathrm{ng} \mathrm{ml}^{-1}$ )-induced CREB phosphorylation was impaired in TrkB ${ }^{\text {GFAP }}$ KO Müller cells. For each determination, the ratio of $p C R E B / C R E B$ protein level in controls (WT, BDNF 0 min) was normalized to a value of 1.0. Data are shown as the mean + s.e.m. $(n=3)$. $(\mathbf{c})$ Immunohistochemical analysis of $p C R E B$ in BDNF $\left(1 \mu g \mu l^{-1}\right)$-treated WT and TrkB GFAP KO retinas. Note the absence of $p C R E B$ in Müller cells (red arrowhead) of the TrkB GFAP $\mathrm{KO}$ retina, despite its persistence in the GCL (arrow). Scale bar, $50 \mu \mathrm{m}$. (d) Effect of BDNF (100 $\mathrm{ng} \mathrm{ml}^{-1}$ for $48 \mathrm{~h}$ ) on trophic factor production in cultured Müller cells from WT and TrkB ${ }^{\text {GFAP }}$ KO mice. For each determination, the mRNA level in controls (WT, without BDNF) was normalized to a value of 1.0. Data are shown as the mean + s.e.m. $(n=6)$. ${ }^{\star} P<0.05$. NGF, nerve growth factor; NT-3, neurotrophin-3; NT-4, neurotrophin-4; CNTF, ciliary neurotrophic factor; GNDF, glial cell line-derived neurotrophic factor.

(Fig. 6b,c). In addition, BDNF significantly increased the expression level of CaMKII $\delta$ but not CaMKII $\alpha$, CaMKII $\beta$ or CaMKII $\gamma$ in WT Müller cells (Fig. 6d). The BDNF-induced increase of CaMKII $\delta$ was not detected in TrkB ${ }^{\mathrm{GFAP}} \mathrm{KO}$ Müller cells (Fig. 6d). In contrast, platelet-derived growth factor, which can also activate CaMKII (ref. 31), increased nestin and Mash1 expression in both WT and TrkB ${ }^{\text {GFAP }}$ KO Müller cells (Fig. 6e,f). These results suggest that BDNF signalling through TrkB stimulates the CaMKII $\delta$-Mash1 pathway, which induces the potential capacity of Müller glia as intrinsic retinal progenitor cells.

\section{Discussion}

In the present study, we used $\operatorname{TrkB}^{\mathrm{GFAP}}$ and $\operatorname{TrkB}{ }^{\mathrm{c}-\mathrm{kit}} \mathrm{KO}$ mice to explore TrkB functions in retinal Müller glia and inner retinal neurons, respectively. We observed that the extent of glutamate-induced retinal degeneration was almost equal in these two mutant mouse models. In addition, BDNF failed to prevent photoreceptor degeneration in $\mathrm{TrkB}^{\mathrm{GFAP}} \mathrm{KO}$ mice. These findings represent the first direct evidence for a role of neurotrophin receptors in glial cells during retinal degeneration. Our results are consistent with the previous 
a

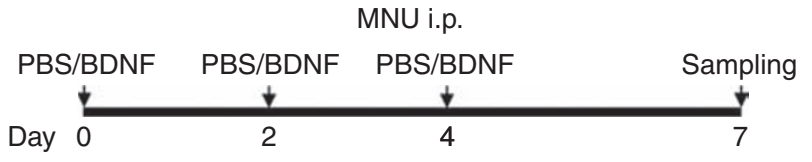

b

$\frac{\text { WT }}{\text { PBS }}$ BDNF $\frac{\text { TrkB }^{\text {GFAP }} \text { KO }}{\text { PBS }}$

$\left(\mathrm{mg} \mathrm{kg}^{-1}\right)$

0

7.5
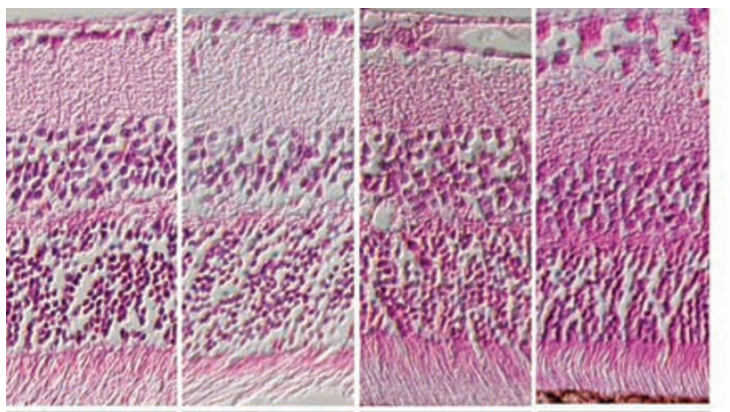

\section{5}

15

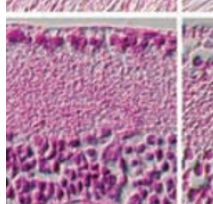

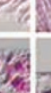
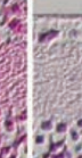

C

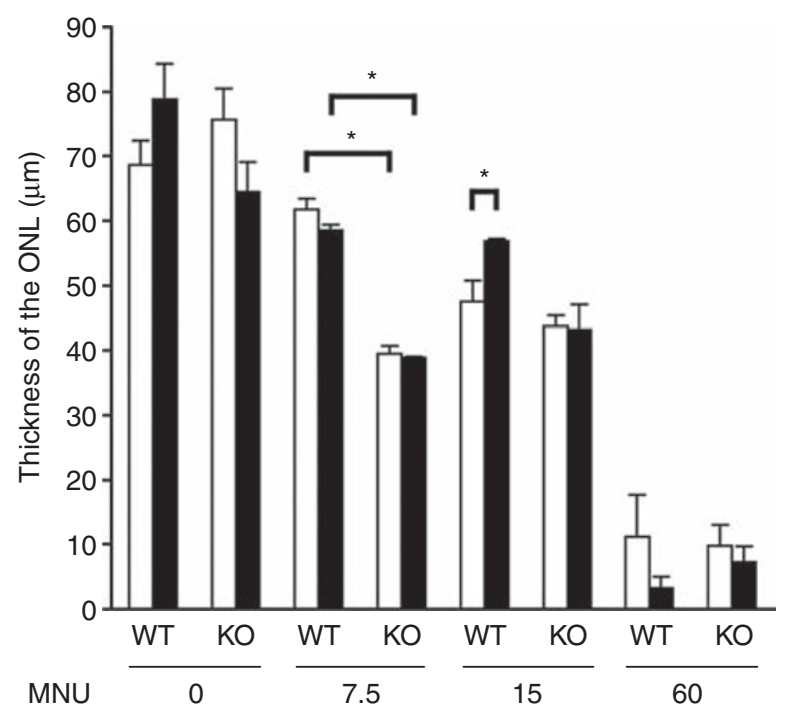

(mg kg-1)

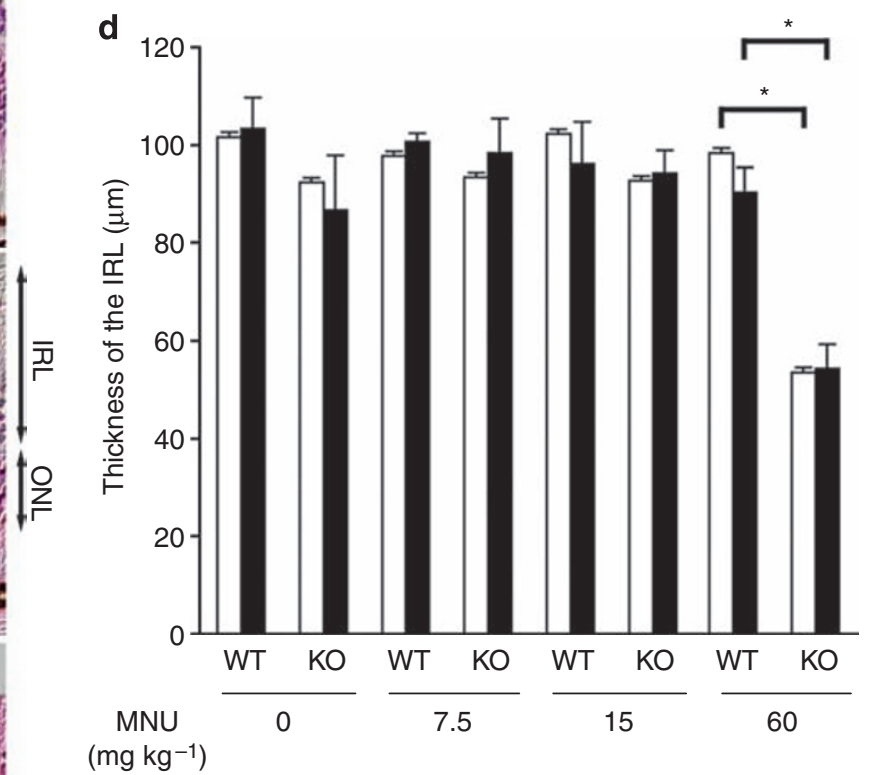

Figure 4 | Accelerated photoreceptor degeneration in TrkB ${ }^{\text {GFAP }}$ KO mice. (a) Animal protocols for MNU-induced photoreceptor degeneration. MNU was injected intraperitoneally (i.p.) into WT and TrkBGFAP KO mice at the concentration of $0,7.5,15$ or $60 \mathrm{mg} \mathrm{kg}^{-1}$. PBS or BDNF $\left(1 \mu \mathrm{g} \mu \mathrm{I}^{-1}\right)$ was intraocularly injected at day 0, 2 and 4, and the animals were killed at day 7 after MNU treatment. (b) Representative photomicrographs of PBS- or BDNF-treated retinas from WT and TrkBGFAP KO mice administered with various concentrations of MNU. Scale bar, $50 \mu \mathrm{m}$. (c, d) Quantitative analysis of the thickness of the ONL (c) and the IRL (d) in PBS (white bar)- or BDNF (black bar)-treated retinas. Data are shown as the mean + s.e.m. $(n=4) .{ }^{*} P<0.05$. IRL, inner retinal layer; ONL, outer nuclear layer.

assumption that the glia-neuron network is heavily involved in the protection of surrounding neurons in $v_{i v o^{12-14,19}}$. Another intriguing result is that BDNF-TrkB signalling stimulated Müller cells to proliferate and express retinal neural cell markers. This finding supports previous work, suggesting that Müller cells have regenerative potential $^{10,11}$. Thus, both BDNF stimulation and intrinsic signal transduction through TrkB may be required for Müller glia to become endogenous retinal progenitor cells. As BDNF induces the production of various trophic factors in Müller cells, including bFGF and insulin growth factor, the regeneration process may involve these trophic factors by an autocrine process. Taken together, our results suggest that neurotrophin signalling in glia is an important mediator of neuroprotection as well as a signalling cue to induce regeneration of radial glia in vivo.

In the retina, glutamate excitotoxicity and oxidative stress may contribute to various eye diseases, including retinal ischaemia and glaucoma ${ }^{8,9,35-38}$. In these disorders, loss of RGCs and subsequent optic nerve degeneration are the primary concerns. As RGCs express Trk receptors, neurotrophins are thought to directly regulate RGC number during development and prevent RGC death in the adult retina ${ }^{34}$. However, our results suggest an unexpectedly powerful neuroprotective effect of TrkB signalling in Müller glia. 
a

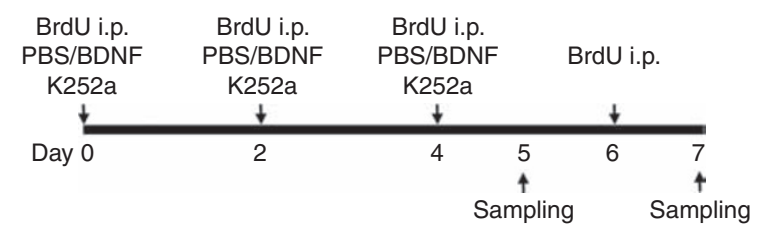

b
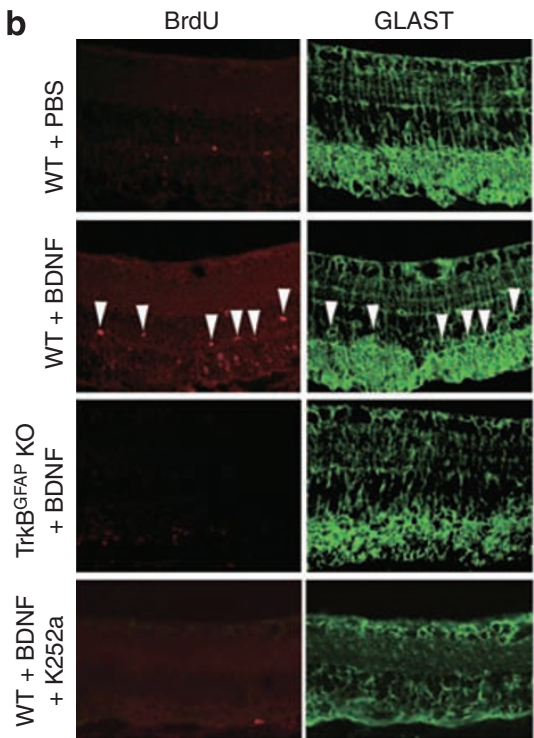

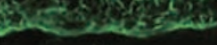
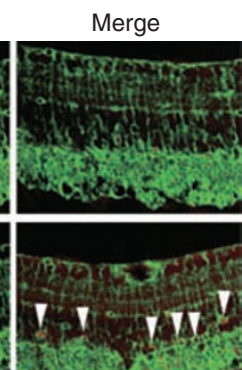

c

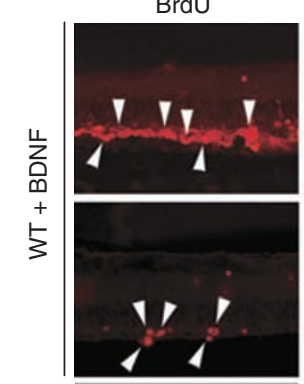

BrdU

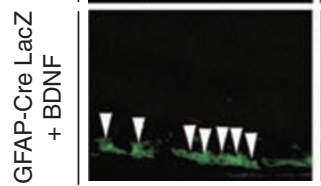

INL d
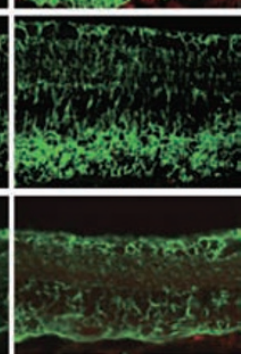

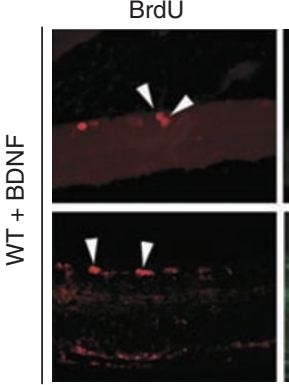

Merge

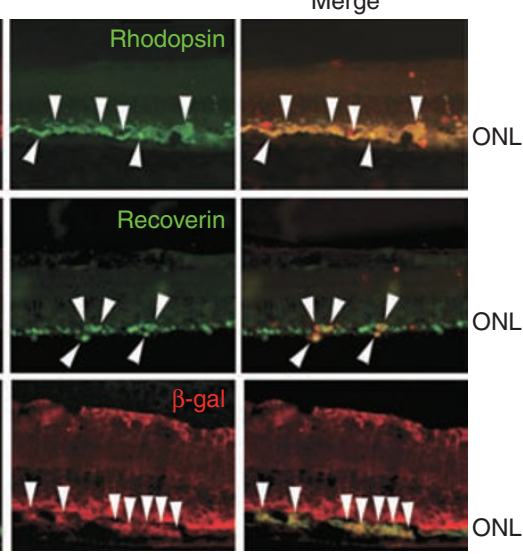

Merge
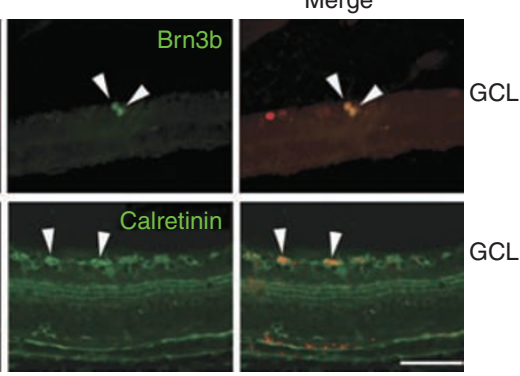

Figure 5 | Effect of BDNF on the proliferation of Müller glial cells during retinal degeneration. (a) Animal protocols. MNU (60 mg kg-1) was injected intraperitoneally (i.p.) to WT, TrkBGFAP KO and GFAP-Cre LacZ mice. BrdU (50 $\mathrm{mg} \mathrm{kg}^{-1}$ ) was injected (i.p.) at day 0, 2, 4 and 6 after MNU treatment. PBS, BDNF $\left(1 \mu \mathrm{g} \mu \mathrm{l}^{-1}\right)$ and K252a (1 mM) were intraocularly injected at day 0, 2 and 4, and the animals were killed at day 5 or 7 . (b) Sections of the retina treated with PBS, BDNF and K252a in WT and TrkB ${ }^{\text {GFAP }}$ KO mice (day 5). BDNF increased BrdU-labelling in GLAST-positive cells in the INL (arrowheads) and some cells in the ONL in WT, but not in TrkBGFAP KO mice. K252a (a blocker for Trk receptors) inhibited BDNF-induced BrdU expression. (c) BrdUlabelled cells in the ONL were double labelled (arrowheads) with rhodopsin or recoverin in WT mice, and with $\beta$-gal in GFAP-Cre LacZ mice (day 7). (d) BrdU-labelled cells in the GCL were double labelled (arrowheads) with Brn3b or calretinin (day 7). Scale bar, $50 \mu \mathrm{m}$. GCL, ganglion cell layer; INL, inner nuclear layer; ONL, outer nuclear layer.

One possible explanation for this phenomenon is that the total neuroprotective effect of Müller glia-derived trophic factors (Fig. 3d) is as effective as that of direct BDNF signalling in RGCs (Fig. 2) (ref. 39). Consistently, transcorneal electrical stimulation, which stimulates Müller cell production of insulin growth factor-1 and BDNF, rescues axotomized RGCs and improves retinal function in human patients ${ }^{40,41}$. Although the number is small compared with Müller glia, we cannot exclude the possibility that astrocytes located in the GCL may also be involved in RGC rescue.

Compared with RGC degeneration, photoreceptor degeneration is a relatively simple disease model in which to explore TrkB function, because $\operatorname{TrkB}$ expression is low in photoreceptors ${ }^{12,13,18}$. In the present study, using the $\operatorname{TrkB}{ }^{\text {GFAP }}$ KO mouse, we demonstrated a functional glia-neuron network during photoreceptor degeneration in vivo. As BDNF increases ciliary neurotrophic factor and glial cell line-derived neurotrophic factor production in WT, but not in TrkBdeficient Müller cells (Fig. 3d), which can directly protect photoreceptors as well as RGCs ${ }^{12,19,27}$, BDNF seems to exert photoreceptor protection by a paracrine mechanism. Consistently, MNU-induced photoreceptor degeneration was observed at a lower dose in TrkB ${ }^{\text {GFAP }}$ KO mice compared with WT mice, and BDNF failed to prevent or delay the degeneration process in the mutant (Fig. 4). Moreover, we found that MNU induced unusual thinning of IRLs in TrkB ${ }^{\text {GFAP }} \mathrm{KO}$ animals (Fig. 4). These results again suggest the importance of glial TrkB signalling for the protection of various types of surrounding neurons during retinal degeneration. One may find that the protective effects of exogenous BDNF observed in our study are small.
As our MNU model is a relatively acute model, it is possible that exogenous BDNF may be more effective in other models that represent milder, slow-progressive photoreceptor degeneration.

We also examined the effect of BDNF on Müller glial cells as intrinsic retinal progenitor cells. We found that BDNF-TrkB signalling stimulated the CaMKII $\delta$-Mash 1 pathway and induced the expression of rod photoreceptor markers in proliferating Müller cells (Figs 5 and 6). This finding is consistent with the previous work that Mash1 is involved in the generation of photoreceptors from retinal progenitor cells ${ }^{33,34}$. A recent study demonstrated that exogenous Wnt3a increases the proliferation of Müller cells in the photoreceptor-damaged adult mouse retina ${ }^{42}$. As Wnt modulates CaMK activity ${ }^{43}$, Wnt signalling may promote adult neurogenesis, at least partly, through the CaMKII $\delta$-Mash1 pathway. In fact, CaMKII activation restored the regeneration capability in the TrkB-deficient Müller cells (Fig. 6e,f). CaMK is a calcium-dependent kinase and activation of CaMK can in turn lead to phosphorylation of $\mathrm{CREB}^{44}$. In our TrkB ${ }^{\mathrm{GFAP}}$ KO mice, BDNF-induced CREB phosphorylation was almost absent in Müller cells, whereas it was clearly detectable in RGCs (Fig. 3c). In astrocytes, TrkB-T1, a truncated form of TrkB that lacks tyrosine kinase activity, may mediate calcium signalling ${ }^{45}$. However, both full-length and truncated forms of TrkB are deleted in our TrkB ${ }^{\text {GFAP }} \mathrm{KO}$ mice $^{25}$, and thus, we have not been able to determine which form of TrkB is necessary for the proliferation and differentiation of Müller cells. Further investigation into subtype-specific TrkB functions in Müller cells may lead to the identification of relevant secondary trophic factors and mechanisms 


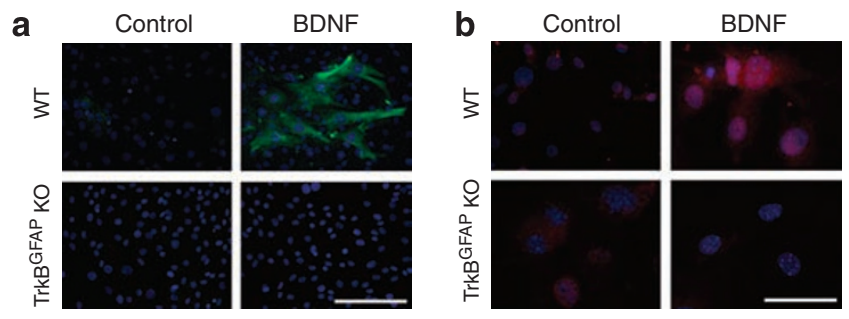

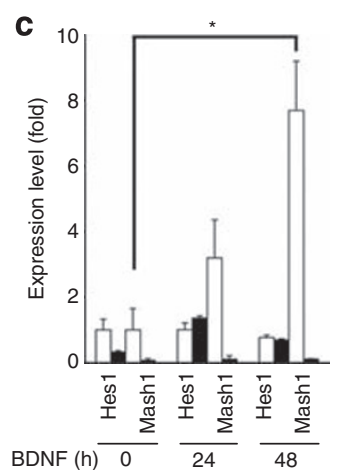

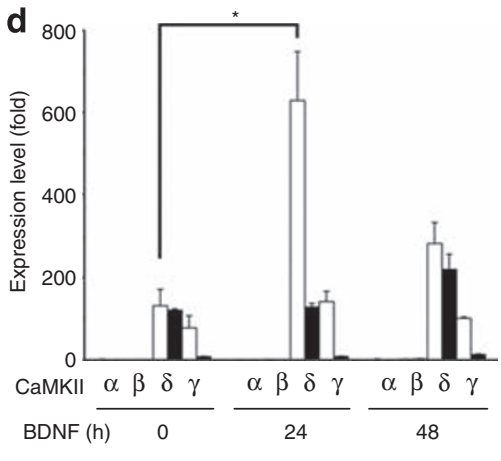

e
Control
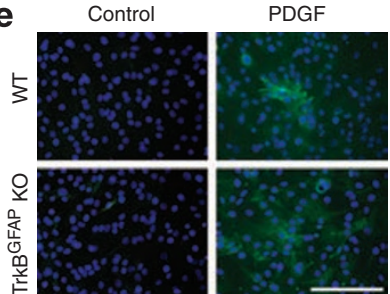

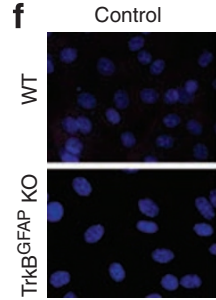

PDGF

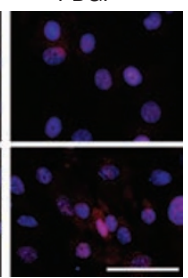

Figure 6 | Regenerative capacity of Müller glial cells mediated by TrkB signalling. (a, b) Treatment with BDNF (100 ng ml-1 for $1-2$ days) induced expression of nestin (a) and Mash1 (b) in cultured Müller cells from WT but not TrkBGFAP KO mice. (c, d) Expression of Hes1 and Mash1 (c) and CaMKII (d) in BDNF-treated Müller cells from WT (white bar) and TrkBGFAP $\mathrm{KO}$ (black bar) mice. Note the upregulation of CaMKII $\delta$ and Mash1 in WT but not TrkB ${ }^{\text {GFAP }}$ KO Müller cells. Data are shown as the mean + s.e.m. $(n=4) .{ }^{\star} P<0.01$. (e, f) Treatment with platelet-derived growth factor (PDGF; $50 \mathrm{ng} \mathrm{ml}^{-1}$ for $24 \mathrm{~h}$ ) induced expression of nestin (e) and Mash1 (f) in cultured Müller cells from WT and TrkB GFAP KO mice. Scale bars, $150 \mu \mathrm{m}$ $(\mathbf{a}, \mathbf{e})$ and $50 \mu \mathrm{m}(\mathbf{b}, \mathbf{f})$.

of effective regeneration in the degenerating retina. We also showed that $\mathrm{BDNF}$ induces the expression of RGC markers in proliferating, that is, BrdU-positive, cells in MNU-treated mice (Fig. 5). However, the rate of occurrence and the number of identified cells were much lower than those for photoreceptor markers. It is possible that degenerating photoreceptors in MNU-treated mice may release trophic factors ${ }^{46}$, including bFGF, which could act in combination with BDNF to stimulate Müller cell proliferation in the ONL. However, this study clearly demonstrates that BDNF-TrkB signalling is one of the active pathways involved in Müller glial cell regeneration. Additional RGC degeneration models and further TrkB signalling and effector pathway studies may lead to better understanding of the mechanisms and transcriptional regulators at play.

In summary, we report important roles of neurotrophin signalling in glial cells as well as in neural cells. Loss of TrkB signalling in glial cells led to severe retinal degeneration in two different animal models. In addition, BDNF-TrkB signalling stimulated the proliferation and differentiation of dedifferentiated Müller glial cells in the degenerating retina. Our glia- and neuron-specific KO mice offer a powerful system for investigating region-specific functions of target genes as well as their contributions to the glia-neuron network in vivo. Recent studies have shown that the unprocessed precursor forms of neurotrophin family members, for example, proBDNF, are released from neurons during development and in adulthood, and such molecules can act through a co-receptor system of $\mathrm{p} 75^{\mathrm{NTR}}$ and sortilin $^{47,48}$. Thus, further study is required to determine whether Müller glia-derived BDNF includes proBDNF and, if so, whether proBDNF induces neural cell apoptosis or inhibits the regenerative potential of Müller cells in vivo.

\section{Methods}

Mice. Experiments were performed using TrkB ${ }^{\text {flox/flox}} ; G F A P-C r e+\left(\operatorname{TrkB}{ }^{\mathrm{GFAP}} \mathrm{KO}\right)$ and $\operatorname{TrkB}^{\text {flox/llox }} ; c$-kit-Cre $+\left(\operatorname{TrkB}^{\text {c-kit }} \mathrm{KO}\right)$ mice $^{22,24,25}$ in accordance with the Tokyo Metropolitan Institute for Neuroscience Guidelines for the Care and Use of Animals. Light intensity inside the cages ranged from 100 to 200 lux under a $12 \mathrm{~h}$ light $/ 12 \mathrm{~h}$ dark cycle.

Histological and morphometric studies. Paraffin sections ( $7 \mu \mathrm{m}$ thick) were cut through the optic nerve and stained with hematoxylin and eosin. The sections were examined with a Microscope (BX51; Olympus) equipped with Plan Fluor objectives connected to a DP70 camera (Olympus). The number of cells in the GCL was counted from one ora serrata through the optic nerve to the other ora serrata ${ }^{37}$. The thickness of the ONL and IRL (between the internal limiting membrane and the interface of the outer plexiform layer and INL) was measured using NIH Image (ImageJ 1.38) (ref. 12). Immunohistochemistry was performed using a Rabbit antibody against TrkB (1:200, Santa Cruz) and a Mouse antibody against GS (1:500, Chemicon) or Calretinin (1:500, Chemicon).

$\boldsymbol{\beta}$-gal expression in GFAP-Cre LacZ and c-kit-Cre LacZ mice. Frozen retinal sections were incubated in X-gal solution at $37^{\circ} \mathrm{C}$ for $12 \mathrm{~h}$ (ref. 23). For double-labelling immunohistochemistry, the sections were incubated with a Goat polyclonal antibody against $\beta$-gal (1:1,000, Biogenesis) and a Mouse antibody against GS (1:500, Chemicon), Calretinin (1:500, Chemicon), PKC (1:500, Sigma), Calbindin (1:100, Sigma) or TUJ1 (1:100, R\&D).

Retinal explant culture. The neural retina without pigment epithelium was placed on a Millicell chamber filter (30-mm diameter, pore size $0.4 \mathrm{~mm}$; Millipore) with the GCL upwards. The chambers were transferred to a 6-well culture plate, with each well containing $1 \mathrm{ml}$ of DMEM/F-12 (Invitrogen) containing 20\% Heat-inactivated horse serum (Invitrogen), changed every other day. The cells were cultured at $34^{\circ} \mathrm{C}$ in $5 \% \mathrm{CO}_{2}$. Retinal explant cultures were incubated with or without $5 \mathrm{mM}$ glutamate for $1 \mathrm{~h}$. After $72 \mathrm{~h}$, retinal explants were immunostained with Mouse anti-NeuN (1:1,000, Chemicon), and the number of NeuN-positive cells in the GCL was quantified ${ }^{49}$.

Müller cell proliferation during photoreceptor degeneration. MNU was injected intraperitoneally (i.p.) into WT, TrkB ${ }^{\mathrm{GFAP}} \mathrm{KO}$ and GFAP-Cre LacZ mice at the concentration of 7.5, 15 or $60 \mathrm{mg} \mathrm{kg}^{-1}$. BrdU was injected (i.p.) at a dose of $50 \mathrm{mg} \mathrm{kg}^{-1}$ at day $0,2,4$ and 6 after MNU treatment. BDNF $\left(1 \mu \mathrm{g} \mu \mathrm{l}^{-1}\right)$ and K252a ( $1 \mathrm{mM}$, Alomone Labs) were intraocularly injected at day 0, 2 and 4. At day 5 or 7 , the animals were killed, and retinal sections were stained with Mouse anti-BrdU (1:20, Roche) and Rabbit anti-GLAST $(1: 1,000)^{8}$, Rabbit anti-Recoverin $(1: 1,000$, Millipore), Goat anti- $\beta$-gal (1:1,000, Biogenesis) or Goat anti-Brn3b (1:200, Santa Cruz) antibodies. Some sections were stained with Rat anti-BrdU (1:40, Abcam) and Mouse anti-Rhodopsin (1:1,000, Sigma) or Mouse anti-Calretinin (1:500, Chemicon) antibodies. The number of BrdU-positive or BrdU/rhodopsin-double positive cells was counted from one ora serrata through the optic nerve to the other ora serrata ${ }^{37}$.

Müller cell culture. Primary Müller cells ${ }^{19}$ were treated with $50 \mathrm{ng} \mathrm{ml}^{-1}$ of BDNF for $0-0.5,12$ or $24 \mathrm{~h}$, and $50 \mathrm{ng} \mathrm{ml}^{-1}$ of platelet-derived growth factor for $24 \mathrm{~h}$. Immunocytochemical analysis was performed with Mouse anti-Nestin (1:100, Chemicon) and Rabbit anti-Mash1 (1:40, R\&D) antibodies, followed by 4,6-diamidino-2-phenylindole staining. Total RNA was extracted with Isogen (Nippon Gene) according to the manufacturer's protocol. Resultant RNA was treated with DNase (RQ1 RNase-Free DNase; Promega) and reverse-transcribed with Revertra ace (Toyobo) to obtain cDNA. Quantitative reverse transcription PCR was performed using the ABI 7500 fast real-time PCR system with SYBR Green PCR Master Mix (Applied Biosystems) ${ }^{50}$. Thermo cycling of each reaction was performed with each primer at a concentration of $100 \mathrm{nM}$. The following protocol was used: denaturation programme $\left(95^{\circ} \mathrm{C}\right.$ for $\left.3 \mathrm{~min}\right)$, followed by the amplification and quantification programme $\left(95^{\circ} \mathrm{C}\right.$ for $15 \mathrm{~s}$ and $60^{\circ} \mathrm{C}$ for $\left.30 \mathrm{~s}\right)$ repeated for 50 cycles. The PCR quality and specificity were verified by melting curve analysis. A standard curve of cycle thresholds using serial dilutions of cDNA samples were used to calculate the relative abundance. The difference in the initial amount of total RNA between the samples was normalized in every assay using glyceraldehyde-3phosphate dehydrogenase $(G A P D H)$ gene expression as an internal standard. The primer probe pairs used are listed in Supplementary Table S1. 
Immunoblotting. Cultured Müller cells were homogenized in ice-cold $50 \mathrm{mM}$ Tris- $\mathrm{HCl}$ (pH 7.4) containing $150 \mathrm{mM} \mathrm{NaCl}, 0.1 \%$ Triton X-100 and a protease inhibitor cocktail (Roche). Protein concentrations were determined using a Bio-Rad protein assay kit (Bio-Rad). Samples were separated on SDS polyacrylamide gel electrophoresis gels and subsequently electrotransferred to Immobilon-P filter (Millipore). Membranes were incubated with Rabbit antibodies against TrkB (1:200, Santa Cruz), Tubulin (1:1,000, Cell Signaling), CREB (1:1,000, Cell Signaling) or pCREB (1:1,000, Cell Signaling). Primary antibody binding was detected using horseradish peroxidase-labelled anti-Mouse IgG secondary antibody (Amersham) and visualized using ECL Plus western blotting system (Amersham)

Statistical analyses. Data are presented as means \pm s.e.m. For statistical analyses, a two-tailed Student's $t$-test was used. $P<0.05$ was regarded as statistically significant.

\section{References}

1. Miller, G . Neuroscience. The dark side of glia. Science 308, 778-781 (2005).

2. Stellwagen, D. \& Malenka, R. C. Synaptic scaling mediated by glial TNF-alpha. Nature 440, 1054-1059 (2006)

3. Barres, B. A. The mystery and magic of glia: a perspective on their roles in health and disease. Neuron 60, 430-440 (2008).

4. Campbell, K. \& Götz, M. Radial glia: multi-purpose cells for vertebrate brain development. Trends Neurosci. 25, 235-238 (2002).

5. Alvarez-Buylla, A. \& Lim, D. A. For the long run: maintaining germinal niches in the adult brain. Neuron 41, 683-686 (2004).

6. Tanaka, E.M. \& Ferretti, P. Considering the evolution of regeneration in the central nervous system. Nat. Rev. Neurosci. 10, 713-723 (2009).

7. Matsugami, T. R. et al. Indispensability of the glutamate transporters GLAST and GLT1 to brain development. Proc. Natl Acad. Sci. USA 103, 12161-12166 (2006).

8. Harada, T et al. Functions of the two glutamate transporters GLAST and GLT-1 in the retina. Proc. Natl Acad. Sci. USA 95, 4663-4666 (1998).

9. Harada, T. et al. The potential role of glutamate transporters in the pathogenesis of normal tension glaucoma. J. Clin. Invest. 117, 1763-1770 (2007).

10. Ooto, S. et al. Potential for neural regeneration after neurotoxic injury in the adult mammalian retina. Proc. Natl Acad. Sci. USA 101, 13654-13659 (2004).

11. Lamba, D., Karl, M. \& Reh, T. Neural regeneration and cell replacement: a view from the eye. Cell Stem Cell 2, 538-549 (2008).

12. Harada, $\mathrm{T}$. et al. Modification of glial-neuronal cell interactions prevents photoreceptor apoptosis during light-induced retinal degeneration. Neuron 26, 533-541 (2000).

13. Wahlin, K. J., Campochiaro, P. A., Zack, D. J. \& Adler, R. Neurotrophic factors cause activation of intracellular signaling pathways in Müller cells and other cells of the inner retina, but not photoreceptors. Invest. Ophthalmol. Vis. Sci. 41, 927-936 (2000).

14. Bringmann, A. et al. Cellular signaling and factors involved in Müller cell gliosis: neuroprotective and detrimental effects. Prog. Retin. Eye Res. 28, 423-451 (2009).

15. Namekata, K. et al. Dock3 induces axonal outgrowth by stimulating membrane recruitment of the WAVE complex. Proc. Natl Acad. Sci. USA 107, 7586-7591 (2010).

16. Blum, R. \& Konnerth, A. Neurotrophin-mediated rapid signaling in the central nervous system: mechanisms and functions. Physiology 20, 70-78 (2005)

17. Nikoletopoulou, V. et al. Neurotrophin receptors TrkA and TrkC cause neuronal death whereas TrkB does not. Nature 467, 59-63 (2010).

18. Grishanin, R. N. et al. Retinal TrkB receptors regulate neural development in the inner, but not outer, retina. Mol. Cell Neurosci. 38, 431-443 (2008)

19. Harada, T. et al. Microglia-Müller glia cell interactions control neurotrophic factor production during light-induced retinal degeneration. J. Neurosci. 22, 9228-9236 (2002)

20. Zhuo, L. et al. hGFAP-cre transgenic mice for manipulation of glial and neuronal function in vivo. Genesis 31, 85-94 (2001).

21. Malatesta, P. et al. Neuronal or glial progeny: regional differences in radial glia fate. Neuron 37, 751-764 (2003).

22. Soriano, P. Generalized lacZ expression with the ROSA26 Cre reporter strain. Nat. Genet. 21, 70-71 (1999).

23. Zhu, Y. et al. Inactivation of NF1 in CNS causes increased glial progenitor proliferation and optic glioma formation. Development 132, 5577-5588 (2005).

24. Eriksson, B., Bergqvist, I., Eriksson, M. \& Holmberg, D. Functional expression of Cre recombinase in sub-regions of mouse CNS and retina. FEBS Lett. 479, 106-110 (2000).

25. Luikart, B. W. et al. TrkB has a cell-autonomous role in the establishment of hippocampal Schaffer collateral synapses. J. Neurosci. 25, 3774-3786 (2005).

26. Rocha, M., Martins, R. A. \& Linden, R. Activation of NMDA receptors protects against glutamate neurotoxicity in the retina: evidence for the involvement of neurotrophins. Brain Res. 827, 79-92 (1999).
27. LaVail, M. M. et al. Multiple growth factors, cytokines, and neurotrophins rescue photoreceptors from the damaging effects of constant light. Proc. Natl Acad. Sci. USA 89, 11249-11253 (1992).

28. Lavail, M. M. et al. Sustained delivery of NT-3 from lens fiber cells in transgenic mice reveals specificity of neuroprotection in retinal degenerations. J. Comp. Neurol. 511, 724-735 (2008).

29. Wenzel, A., Grimm, C., Samardzija, M. \& Remé, C. E. Molecular mechanisms of light-induced photoreceptor apoptosis and neuroprotection for retinal degeneration. Prog. Retin. Eye Res. 24, 275-306 (2005).

30. Yoshizawa, K. \& Tsubura, A. Characteristics of $N$-methyl- $N$-nitrosoureainduced retinal degeneration in animals and application for the therapy of human retinitis pigmentosa. Nippon Ganka Gakkai Zasshi 109, 327-337 (2005).

31. Ju, B. G. et al. Activating the PARP-1 sensor component of the groucho/TLE1 corepressor complex mediates a CaMKII $\delta$-dependent neurogenic gene activation pathway. Cell 119, 815-829 (2004).

32. Parras, C. M. et al. Mash1 specifies neurons and oligodendrocytes in the postnatal brain. EMBO J. 23, 4495-4505 (2004).

33. Tomita, K., Nakanishi, S., Guillemot, F. \& Kageyama, R. Mash1 promotes neuronal differentiation in the retina. Genes Cells 1, 765-774 (1996).

34. Harada, T., Harada, C. \& Parada, L. F. Molecular regulation of visual system development: more than meets the eye. Genes Dev. 21, 367-378 (2007)

35. Lipton, S. A. Retinal ganglion cells, glaucoma and neuroprotection. Prog. Brain Res. 131, 712-718 (2001).

36. Ohia, S. E., Opere, C. A. \& LeDay, A. M. Pharmacological consequences of oxidative stress in ocular tissues. Mutation Res. 579, 22-36 (2005).

37. Harada, C. et al. Role of apoptosis signal-regulating kinase 1 in stress-induced neural cell apoptosis in vivo. Am. J. Pathol. 168, 261-269 (2006).

38. Harada, C. et al. ASK1 deficiency attenuates neural cell death in GLASTdeficient mice, a model of normal tension glaucoma. Cell Death Differ. 17, 1751-1759 (2010).

39. Johnson, E. C., Guo, Y ., Cepurna, W.O. \& Morrison, J. C. Neurotrophin roles in retinal ganglion cell survival: lessons from rat glaucoma models. Exp. Eye Res. 88, 808-815 (2009).

40. Morimoto, T. et al. Transcorneal electrical stimulation rescues axotomized retinal ganglion cells by activating endogenous retinal IGF-1 system. Invest. Ophthalmol. Vis. Sci. 46, 2147-2155 (2005).

41. Inomata, K . et al. Transcorneal electrical stimulation of retina to treat longstanding retinal artery occlusion. Graefes Arch. Clin. Exp. Ophthalmol. 245, 1773-1780 (2007)

42. Osakada, F. et al. Wnt signaling promotes regeneration in the retina of adult mammals. J. Neurosci. 27, 4210-4219 (2007).

43. Inestrosa, N. C. \& Arenas, E. Emerging roles of Wnts in the adult nervous system. Nat. Rev. Neurosci. 11, 77-86 (2010).

44. Ernfors, P. \& Bramham, C. R. The coupling of a trkB tyrosine residue to LTP. Trends Neurosci. 26, 171-173 (2003).

45. Rose, C. R. et al. Truncated TrkB-T1 mediates neurotrophin-evoked calcium signalling in glia cells. Nature 426, 74-78 (2003)

46. Faktorovich, E. G., Steinberg, R. H., Yasumura, D., Matthes, M. T. \& LaVail, M. M. Basic fibroblast growth factor and local injury protect photoreceptors from light damage in the rat. J. Neurosci. 12, 3554-3567 (1992).

47. Yang, J. et al. Neuronal release of proBDNF. Nat. Neurosci. 12, 113-115 (2009).

48. Lee, R., Kermani, P., Teng, K. K. \& Hempstead, B. L. Regulation of cell survival by secreted proneurotrophins. Science 294, 1945-1948 (2001).

49. Namekata, K., Harada, C., Kohyama, K., Matsumoto, Y. \& Harada, T. Interleukin-1 stimulates glutamate uptake in glial cells by accelerating membrane trafficking of $\mathrm{Na}^{+} / \mathrm{K}^{+}$-ATPase via actin depolymerization. Mol. Cell Biol. 28, 3273-3280 (2008)

50. Guo, X. et al. Inhibition of glial cell activation ameliorates the severity of experimental autoimmune encephalomyelitis. Neurosci. Res. 59, 457-466 (2007)

\section{Acknowledgments}

We thank A. Messing for providing GFAP-Cre mice, B. Eriksson for providing c-kit-Cre mice, R. Shimizu and M. Kasuya for technical assistance, and R. McKay for comments on the manuscript. This work was supported by the Ministry of Education, Culture, Sports, Science and Technology of Japan (C.H., X.G., K. Namekata, T.H.), the Ministry of Health, Labour and Welfare of Japan (K.T., T.H.), Japanese Retinitis Pigmentosa Society, the Japan Medical Association (T.H.), the National Institute of Neurological Disorders and Stroke, the American Cancer Society, and the Department of Defense (L.F.P.)

\section{Author contributions}

C.H., L.F.P. and T.H. designed the study and wrote the paper. K.Namekata and K.T contributed to the study design. C.H., X.G., K.Namekata, A.K., K.Nakamura and T.H. conducted the experiments. All authors interpreted the data 


\section{Additional information}

Supplementary Information accompanies this paper at http://www.nature.com/ naturecommunications

Competing financial interests: The authors declare no competing financial interests.

Reprints and permission information is available online at http://npg.nature.com/ reprintsandpermissions/
How to cite this article: Harada, C. et al. Glia- and neuron-specific functions of TrkB signalling during retinal degeneration and regeneration. Nat. Commun. 2:189 doi: 10.1038/ncomms1190 (2011).

License: This work is licensed under a Creative Commons Attribution-NonCommercialShare Alike 3.0 Unported License. To view a copy of this license, visit http:// creativecommons.org/licenses/by-nc-sa/3.0/ 